\title{
A numerical solution of the dispersion equation of the guided wave propagation in N-layered media
}

\author{
Juan Cardona $^{1}$, Pedro Tabuenca ${ }^{2}$ and Avelino Samartín ${ }^{3}$ \\ 1 Dept. of Electrical Engineering and Energetic, Univ. of Cantabria, cardonaj@unican.es \\ 2 Dept. of Applied Physics, University of Cantabria, tabuencp@ orange.es \\ 3 Dept. of Structural Mechanics, Technical University of Madrid, avelino.samartin@upm.es
}

July 8, 2008

Keywords: Ultrasonic waves, Guided waves, Multilayer media, composite materials, dispersion curves, numerical techniques

\begin{abstract}
The theory of guided wave propagation in N-layered media is presented. The derivation of the dispersion equation is obtained from the application of appropriate boundary and continuity conditions to the solution of the general wave propagation. The resulting dispersion equation is given in the form of a determinant of a $4 \mathrm{Nx} 4 \mathrm{~N}$ coefficient matrix. A numerical procedure is proposed to represent and solve the implicit equation resulting. The validity and efficiency of the proposed numerical model is discussed. Dispersion curves characterizing the $\mathrm{N}$-layered material are obtained and compared to published results. In order to illustrate the use of the model to different practical applications, such as coating problems of plasma spray on a turbine blade, aircraft multiple layers, ice detection, etc. a sensitivity analysis of the dispersion curves respect to depth imperfections is given.
\end{abstract}


Finally, the model can be extended to compute displacements, stresses and strain fields in the layered media.

\section{Introduction}

The study of wave propagation through elastic solid media can be used to carry out non-destructive tests (NDT) of structures. These tests can be used to detect and identify, in many cases, both the actual elastic properties and possible geometric imperfections included material damage of a structure. Typically, a static test of a structure presents a local character, contrarily to a dynamic one that excites the whole structure. In the case of low-frequency vibrations, as the solid body waves, it is possible to discover and characterize material properties and geometry of the structure, by studying the natural modes and frequencies of the different waves propagation. However, if in case of high-frequency guided waves the study of a single wave sometimes reveal the whole nature of the structure being tested.

One important application of high-frequency waves is the characterization of composite materials.

A composite material consists of several thin layers or laminae and the resultant solid acts as a full plate. The layers can be of different materials, but typically the same material is used across the plate. In many cases, if the layer material is orthotropic, the layers are arranged with different orientation and as a consequence the structural behavior of composite material differs according to the sequence of orientations.

In the framework of Materials Science one important issue corresponds to the design and estimation of the mechanical properties of a composite material. The design of a composite material consists in define for each laminate its thickness and orientation. However, prior to a design, an effective method of analysis has to be developed. More detailed studies on the mechanics of composite materials can be found in the existing extensive literature [3], [9], [11], [10]. A main goal in the design of composite materials is optimize the component laminae thicknesses and orientations to be used in a project. This subject of composite materials optimal design is of great interest and it has been treated in [2].

In order to check the accuracy of a composite material design a NDT is usually carried out. The application of high-frequency wave propagation theory is often considered to reach this objective. In this paper the general theory of highfrequency wave propagation in layered media will be summarized and the dispersion equation is obtained. The dispersion equation, as a result of the solution of a generalized nonlinear eigenvalue and eigenvector problem, is found. Special emphasis on the numerical methods to effectively solve this nonlinear eigenvector 
problem will be given and some of them will be described.

Before to develop the general theory of wave propagation in a $\mathrm{N}$-layered medium it is convenient to introduce the simple case of $N=1$, i.e, the Lamb wave propagation. That is presented in section 2 . The dispersion curves of this case are simple and can be used in order to be compared with the results found from the general theory of a multi-layered body described in section 4. Finally, in the paper two remaining section a sensitivity analysis are given and some conclusions are reached.

\section{Lamb guided waves}

Lamb waves can be used to damage detection of partially penetrating cracks appearing in a plate. The detection of these cracks is important as they are related to structural safety. However, partially penetrating cracks are usually very difficult to observe, because they may not appear on the free faces of the plate. An excellent summary of the general theory of wave propagation is given in [4]. A more detailed description is presented in the recent texts [7] [6]. Here only final results are shown. They will be used later in a model and a numerical analysis of these surface waves.

Lamb waves corresponds a particular case of propagation of elastic waves throughout an infinite solid that appears when the infinite solid becomes an infinite plate bounded by two free parallel faces. In this case, very often waves reflections along the faces of the plate occur and therefore the propagation of the waves modifies its direction. The wave propagation in this situation is known as guided waves.

It is assumed an homogenous and isotropic elastic plate bounded by two parallel planes separated a small distance $2 h$. In figure 1 the plate and the adopted coordinate axes are shown. Then $x_{2}= \pm h$ are the equations of the plate free faces and the plane $\left(x_{1}, x_{2}\right)$, containing the normal $x_{3}$ and the direction of the wave propagation, is called sagittal plane.

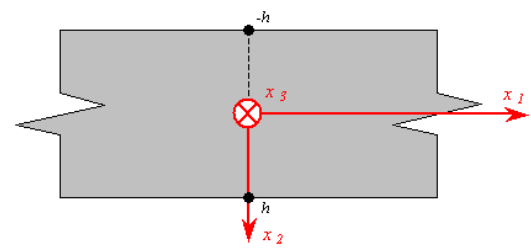

Figure 1: Isotropic plate. Coordinate axes

In the case of a thin plate in which longitudinal (L) and transversal vertical 
(TV) waves are propagated in its vertical plane $\left(x_{1}, x_{2}\right)$ with successive reflections on its free surfaces $\pm h$,i. e. in this propagation there exist a coupling in material displacements with these planes. These waves are called guided waves. However, transversal $(\mathrm{TH})$ waves contained in the horizontal plane $x_{2}$ are propagating only in the horizontal plane of the plate ${ }^{1}$, because their polarization is not modified by eventual reflections and refractions. These guided waves are known as Lamb waves and they can be classified as follows: The first waves, that are propagating in the sagittal plane $\left(x_{1}, x_{2}\right)$ are the Lamb waves $\mathrm{L}_{2}$ and the uncoupled and polarized waves in the plane $\left(x_{1}, x_{3}\right)$ are the Lamb TH waves.

In the following guided normal waves Lamb $\mathrm{L}_{2}$ will be considered. Theses waves appear in a plate of thickness $2 h$ comparable to the wave length, due to the existing coupling between the longitudinal $\mathrm{L}$ and transverse TV wave components. In this way, two types of Lamb waves can be produced. The symmetric waves (Figure 2), in which on either side of the middle plane of the plate, the longitudinal components are equal and the transverse components are opposite and the antisymmetric waves (Figure 3 ) in which on either side of the middle plane of the plate the transverse components are equal and the longitudinal ones opposite. The Rayleigh waves $R_{2}$, to be used in the next section, are related to the Lamb waves. They are produced when the plate thickness $2 h$ is much greater than the wave length and they are propagated along the free boundary and independently of the plate thickness.

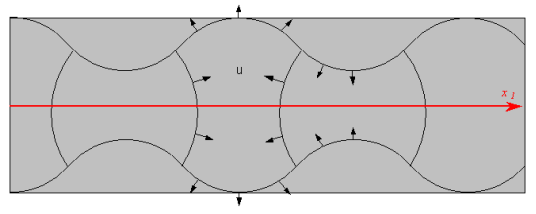

Figure 2: Lamb waves. Symmetric mode

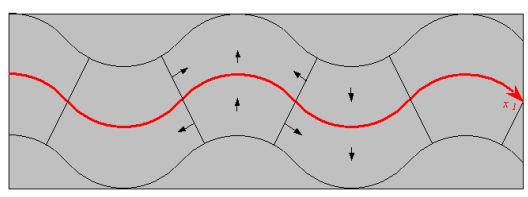

Figure 3: Lamb waves. Antisymmetric mode

\footnotetext{
${ }^{1}$ Similar situation occurs if a stratified plate is considered instead of the plate with its two faces free as in the text.
} 
According to the general theory of wave propagation, the displacement vector $\mathbf{u}$ of a material point can be derived from a potential scalar $\phi$ and a vector potential $\psi$, so that the following relation holds

$$
\mathbf{u}=\boldsymbol{\nabla} \phi+\nabla \times \psi
$$

In the expression (1) the two potentials should be fulfill the two wave equations

$$
\begin{aligned}
& \nabla^{2} \phi-\frac{1}{v_{L}^{2}} \frac{\partial^{2} \phi}{\partial t^{2}}=0 \quad \text { with } \quad v_{L}=\left(\frac{c_{11}}{\rho}\right)^{\frac{1}{2}} \\
& \nabla^{2} \boldsymbol{\psi}-\frac{1}{v_{T}^{2}} \frac{\partial^{2} \boldsymbol{\psi}}{\partial t^{2}}=0 \quad \text { with } \quad v_{T}=\left(\frac{c_{44}}{\rho}\right)^{\frac{1}{2}}
\end{aligned}
$$

with $v_{L}$ and $v_{T}$ are the phase velocities of the longitudinal and transverse waves. The elastic constants $c_{\alpha \beta}, \alpha, \beta=1,2, \ldots, 6$ are defined as function of the Young modulus $E$ and the Poisson ratio $\nu$ as follows:

$$
\begin{aligned}
& c_{11}=c_{22}=c_{33}=\frac{(1-\nu) E}{(1+\nu)(1-2 \nu)} \\
& c_{12}=c_{23}=c_{13}=\frac{\nu E}{(1+\nu)(1-\nu)} \\
& c_{44}=c_{55}=c_{66}=\frac{E}{2(1+\nu)}=\frac{c_{11}-c_{12}}{2}
\end{aligned}
$$

in which the remaining nonsymmetric terms $c_{\alpha \beta}(\alpha \neq \beta)$ are null.

As it is known the stresses and strains associated to the volume changes can be expressed in terms of the function $\phi$ and the stresses producing only shear deformations, without volume changes, can be expressed in terms of $\psi$.

It is assumed Lamb waves travel along the axis $x_{1}$ and diffraction in the $x_{3}$ is ignored. In the case of an isotropic and homogenous elastic solid the scalar and vector potentials are trigonometric functions of time $t$ with the same frequency $\omega$. Then, they can be expressed in the following way, with $k$ the wave number:

$$
\phi=\phi_{0}\left(x_{2}\right) e^{i\left(\omega t-k x_{1}\right)} \quad \text { and } \quad \boldsymbol{\psi}=\left[\psi_{0 j}\left(x_{2}\right)\right] e^{i\left(\omega t-k x_{1}\right)}, j=1,2,3
$$

A boundary value problem of the waves can be defined by the wave equations for each potential function and the boundary conditions $\sigma_{2 i}=0, i=1,2,3$ on the free faces $x_{2}= \pm h$. In order this boundary value problem can have a non trivial solution, it is necessary that the frequency $\omega$ and the wave number $k$ satisfy the following dispersion relation, called Rayleigh-Lamb equation:

$$
\frac{\omega^{4}}{v_{T}^{4}}=4 k q^{2}\left[1-\frac{p \tan (p h+\alpha)}{q \tan (q h+\alpha)}\right] \quad \text { with } \quad \alpha=0 \text { and } \alpha=\frac{\pi}{2}
$$


where the constants $p$ and $q$ are defined as follows:

$$
p^{2}=\frac{\omega^{2}}{v_{L}^{2}}-k^{2} \quad \text { and } \quad q^{2}=\frac{\omega^{2}}{v_{T}^{2}}-k^{2}
$$

and the angle constant $\alpha$ can take the values 0 and $\frac{\pi}{2}$ depending on the type of symmetry of the Lamb wave, as it will be discussed later.

If the relation (8) is satisfied, then the potencial functions can be found, except by a constant factor and their expressions are:

$\psi_{1}=\psi_{2}=0, \psi_{3}=A \sin \left(q x_{2}+\alpha\right) \exp \left[i\left(\omega t-k x_{1}\right)\right]$ and $\phi=B \cos \left(p x_{2}+\alpha\right) \exp \left[i\left(\omega t-k x_{1}\right)\right]$

in which the constants $A$ and $B$ have to satisfied the following linear homogenous system of simultaneous equations:

$$
\left[\begin{array}{cc}
\left(k^{2}-q^{2}\right) \cos (p h+\alpha) & 2 i k q \cos (q h+\alpha) \\
2 i k p \sin (p h+\alpha) & \left(k^{2}-q^{2}\right) \cos (q h+\alpha)
\end{array}\right]\left[\begin{array}{c}
B \\
A
\end{array}\right]=\left[\begin{array}{l}
0 \\
0
\end{array}\right]
$$

Once the functions $\phi\left(x_{1}, x_{2}, t\right)$ and $\boldsymbol{\psi}\left(x_{1}, x_{2}, t\right)$ are known, the application of formulae (1) gives the displacements, except by a constant factor $A$, at time $t$ of any material point $\left(x_{1}, x_{2}\right)$ of the plate, according to the expressions

$$
\begin{aligned}
& u_{1}=q A\left[\cos \left(q x_{2}+\alpha\right)-\frac{2 k^{2}}{k^{2}-q^{2}} \frac{\cos (q h+\alpha)}{\cos (p h+\alpha)} \cos \left(p x_{2}+\alpha\right)\right] \exp \left[i\left(\omega t-k x_{1}\right)\right] \\
& u_{2}=i k A\left[\sin \left(q x_{2}+\alpha\right)+\frac{2 p q}{k^{2}-q^{2}} \frac{\cos (q h+\alpha)}{\cos (p h+\alpha)} \sin \left(p x_{2}+\alpha\right)\right] \exp \left[i\left(\omega t-k x_{1}\right)\right]
\end{aligned}
$$

The equation (8) can be represented in the plane $(\omega, k)$ and then it defines a curve known as dispersion curve. In this curve three regions can be distinguished, according to the value of the phase velocity $V=\frac{\omega}{k}$ is greater either than the longitudinal wave velocity $v_{L}$ or than the transverse velocity velocity $v_{T}$. Then, the relations (9) can be written as follows:

$$
p^{2}=\omega^{2}\left(\frac{1}{v_{L}^{2}}-\frac{1}{V^{2}}\right), \quad q^{2}=\omega^{2}\left(\frac{1}{v_{T}^{2}}-\frac{1}{V^{2}}\right)
$$

and therefore the following boundaries for the regions of dispersion space can be defined: 
- Región 1.- $V>v_{L}>v_{T}$ or equivalently $k<\frac{\omega}{v_{L}}<\frac{\omega}{v_{T}}$.

The wave numbers $p$ and $q$ are both real.

- Región 2.- $v_{L}>V>v_{T}$ or equivalently $\frac{\omega}{v_{L}}<k<\frac{\omega}{v_{T}}$.

The wave numbers are $q$ real and $p$ imaginary.

- Región 3.- $v_{L}>v_{T}>V$ or equivalently $\frac{\omega}{v_{L}}<\frac{\omega}{v_{T}}<k$.

The wave numbers, $p$ and $q$, are both imaginary.

In the figure 4 the three regions are shown.

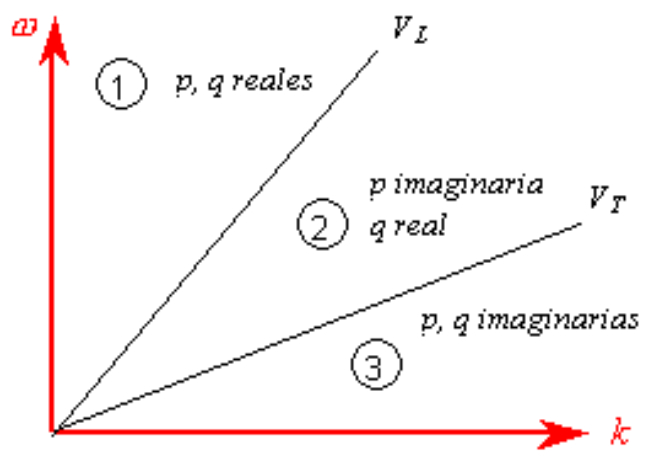

Figure 4: Rayleigh-Lamb dispersion equation. Division of the plane $\omega-k$ in three regions

For each of the three above region the corresponding expressions for the displacements when the real components of the expressions (12) and (13) are considered:

- Región 1 ( $p$ and $q$ are real)

$$
\begin{aligned}
& u_{1}=q A\left[\cos \left(q x_{2}+\alpha\right)-\frac{2 k^{2}}{k^{2}-q^{2}} \frac{\cos (q h+\alpha)}{\cos (p h+\alpha)} \cos \left(p x_{2}+\alpha\right)\right] \cos \left(\omega t-k x_{1}\right) \\
& u_{2}=-k A\left[\sin \left(q x_{2}+\alpha\right)+\frac{2 p q}{k^{2}-q^{2}} \frac{\cos (q h+\alpha)}{\cos (p h+\alpha)} \sin \left(p x_{2}+\alpha\right)\right] \sin \left(\omega t-k x_{1}\right)
\end{aligned}
$$


- Región 2 ( $p$ is imaginary, i.e. $p i$ and $q$ real)

$$
\begin{aligned}
& \text { Case } \alpha=0 \\
& u_{1}=q A\left[\cos q x_{2}-\frac{2 k^{2}}{k^{2}-q^{2}} \frac{\cos q h}{\cosh p h} \cosh p x_{2}\right] \cos \left(\omega t-k x_{1}\right) \\
& u_{2}=-k A\left[\sin q x_{2}-\frac{2 p q}{k^{2}-q^{2}} \frac{\cos q h}{\cosh p h} \sinh p x_{2}\right] \sin \left(\omega t-k x_{1}\right)
\end{aligned}
$$

Case $\alpha=\frac{\pi}{2}$

$$
\begin{aligned}
& u_{1}=q A\left[-\sin q x_{2}+\frac{2 k^{2}}{k^{2}-q^{2}} \frac{\sin q h}{\sinh p h} \sinh p x_{2}\right] \cos \left(\omega t-k x_{1}\right) \\
& u_{2}=-k A\left[\cos q x_{2}+\frac{2 p q}{k^{2}-q^{2}} \frac{\sin q h}{\sinh p h} \cosh p x_{2}\right] \sin \left(\omega t-k x_{1}\right)
\end{aligned}
$$

- Región 3 ( $p$ and $q$ are imaginary, i.e. $p i$ and $q i$ )

Case $\alpha=0$

$$
\begin{aligned}
& u_{1}=q A\left[\cosh q x_{2}-\frac{2 k^{2}}{k^{2}+q^{2}} \frac{\cosh q h}{\cosh p h} \cosh p x_{2}\right] \cos \left(\omega t-k x_{1}\right) \\
& u_{2}=-k A\left[\sinh q x_{2}-\frac{2 p q}{k^{2}+q^{2}} \frac{\cosh q h}{\cosh p h} \sinh p x_{2}\right] \cos \left(\omega t-k x_{1}\right)
\end{aligned}
$$

Case $\alpha=\frac{\pi}{2}$

$$
\begin{aligned}
& u_{1}=q A\left[\sinh q x_{2}-\frac{2 k^{2}}{k^{2}+q^{2}} \frac{\sinh q h}{\sinh p h} \sinh p x_{2}\right] \sin \left(\omega t-k x_{1}\right) \\
& u_{2}=-k A\left[\cosh q x_{2}-\frac{2 p q}{k^{2}+q^{2}} \frac{\sinh q h}{\sinh p h} \cosh p x_{2}\right] \sin \left(\omega t-k x_{1}\right)
\end{aligned}
$$

A particular case of special interest of wave Lamb corresponds to the values $q^{2}=k^{2}$. Then the equations (9) lead to the results

$$
\frac{\omega^{2}}{v_{T}^{2}}=2 k^{2} \quad \rightarrow \quad V=\frac{\omega}{k}=v_{T} \sqrt{2}
$$

i.e., as the following relations $v_{L}>V>v_{T}$ are satisfied, the dispersion curve is in the region 2, and then $p$ is imaginary $(p=\chi i)$. Moreover the plate velocity $V_{P}$ 
fulfills the condition

$$
V_{P}=v_{T} \sqrt{2} \sqrt{1+\frac{c_{12}}{c_{11}}}>v_{T} \sqrt{2}
$$

where the plate velocity $V_{P}$ is the limit of phase velocity when the frequency $\omega$ and $k$, both approach to zero in a Lamb wave symmetric mode. The equation (16) is valid for every mode except for the antisymmetric mode, represented by $\mathrm{A}_{0}$, with a velocity $V$ smaller than $v_{T}$.
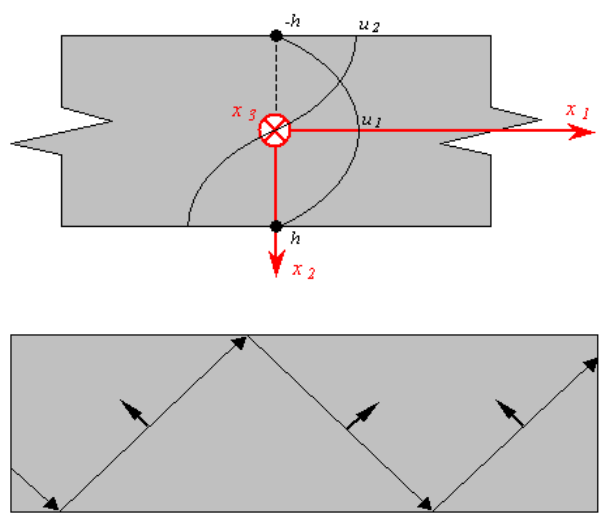

Figure 5: Lamb wave (Lamé mode) for $q^{2}=k^{2}$. (a) Propagation to $45^{\circ}$ (b) Displacements

In general, when $q=k$ it can be written $k h=\frac{n \pi}{2}$ with $n$ odd for symmetric modes and even for antisymmetric modes. The Lamé modes appear for equally spaced values of the frequency-thickness product, given by the formula

$$
2 h f=n \frac{v_{T}}{\sqrt{2}}, \quad n=1,2, \ldots
$$

In figure $5 \mathrm{a}$ the variations of $u_{1}$ and $u_{2}$ throughout the plate thickness for the symmetric mode $\mathrm{S}_{0}$ is shown. The displacement $u_{1}$ is null along the plate faces because the Lamb wave is simply a TV wave, i.e. a transverse wave polarized in the sagittal plane $x_{1} x_{2}$. As it can be observed in figure $5 \mathrm{~b}$ this mode is propagating, along the plate axis $x_{1}$, with an angle $\frac{\pi}{4}$ respect to the axis $x_{1}$, such that $V=v_{T} \sqrt{2}$.

\subsection{Numerical simulation. Application}

A illustrative example of the application of the Lamb waves as a tool to identify partially penetrating cracks in metallic plates an laboratory test will be numerically simulated. 


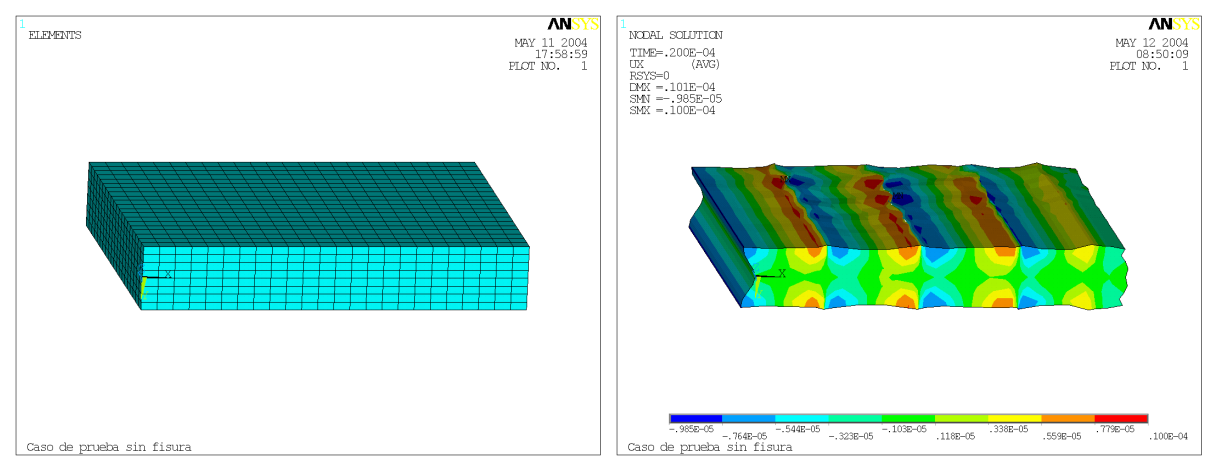

Figure 6: Uncracked plate. (a) FE mesh. (b) Displacements $u_{1}$ at $t=2.5 \times 10^{-5}$ sec

In the following a rectangular steel plate of the following dimensions is considered. Total thickness $2 h=0.02 \mathrm{~m}$, plan dimensions $a=0.200 \mathrm{~m}$ and $b=0.120$ m. A train of symmetric Lamb waves $\left(\alpha=0\right.$ of frequency $f=200 \times 10^{3} \mathrm{~Hz}$ is introduced in the plate trough the side of length $a$. The material of the plate has the following characteristics: Young modulus $E=1.962 \times 10^{8} \mathrm{MPa}$, Poisson ratio $\nu=0.3093$ and density $\rho=7.797 \mathrm{t} / \mathrm{m}^{3}$. From these data the angular frequency is $\omega=1256637.061 \mathrm{rad} / \mathrm{sec}$, the transverse and longitudinal velocities are: $v_{T}=3099.9248 \mathrm{~m} / \mathrm{sec}$ and $v_{L}=5889.5724 \mathrm{~m} / \mathrm{sec}$ and from the dispersion equation the wave number is $k=218.1658 \mathrm{~m}^{-1}$ and the wave velocity is $V=5760.01 \mathrm{~m} / \mathrm{sec}$ are obtained. In this particular case the Lamb wave is situated in the region 2, i.e. $p$ is an imaginary number and $p$ is a real one.

Using the properties of symmetry of the analysis half plate has been modeled. The FE mesh has been uniform with 24 divisions along the longitudinal direction $x_{1}, 8$ divisions along direction $x_{2}$ and 20 through the thickness (direction $x_{3}$ ). The the maximum side length of an element is $0.005 \mathrm{~m}$ and the time increment used was 120 per cycle i.e. $\Delta t=4,17 \times 10^{-8} \mathrm{sec}$. The FE mesh used in the analysis is shown in figure $6 a$.

The displacement results of the wave propagation analysis for a particular time are shown in figure $6 \mathrm{~b}$.

Similar procedure has been carried out on a damaged plate with a crack parallel to side $a$ and situated at section $x_{1}=0.007 \mathrm{~m}$. The total length of the crack was $0.010 \mathrm{~m}$ and its depth was 0.010 . The constant crack width was $0.002 \mathrm{~m}$. The mesh and the results are shown in figures $7 \mathrm{a}$ and $7 \mathrm{~b}$. It can be observed that the dimensions and position of the crack are directly revealed in this analysis ${ }^{2}$.

\footnotetext{
${ }^{2}$ In this preliminary analysis it has not been considered the possible impact-contact between the two faces of the crack
} 


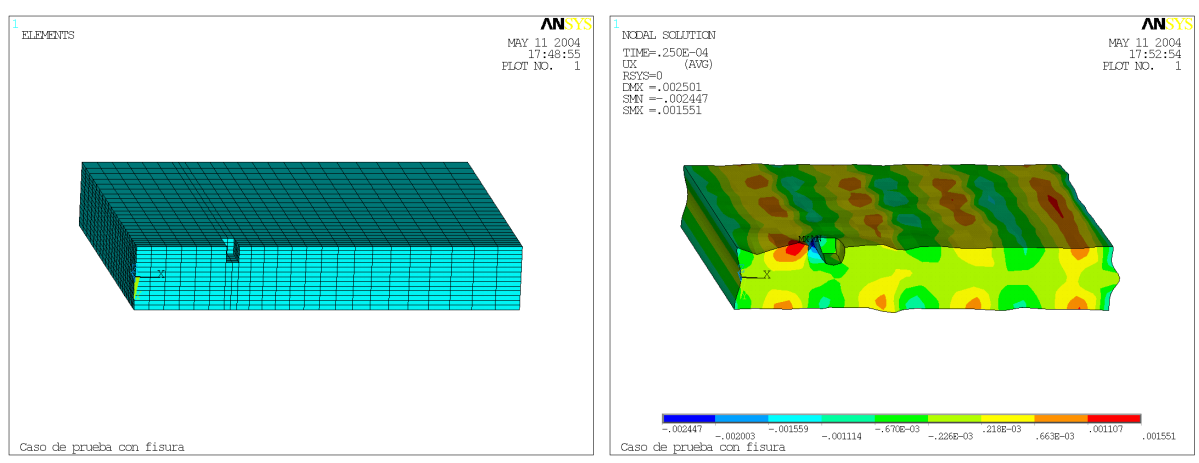

Figure 7: Cracked plate. (a) FE mesh. (b) Displacements $u_{1}$ at $t=2.5 \times 10^{-5} \mathrm{sec}$

\section{Guided waves in N-layered media}

The objective is to obtain the dispersion curves, i.e. to find the wave number $k$ wave for each circular frequency $\omega$ of the wave propagation through an elastic solid of thickness $H$ composed by $N$ layers. From this result it can be obtained the wavelength $\lambda=\frac{2 \pi}{k}$ as well as the phase velocity $c=\frac{\lambda}{T}$, in which the period $T$ is defined by $T=\frac{2 \pi}{\omega}=\frac{1}{f}$ with $f$ the frequency.

\subsection{Hypotheses and notation}

It is considered an infinite elastic solid bounded by two parallel horizontal planes separated a distance $H$. The origin $O$ of cartesian coordinate system $O x_{1} x_{2} x_{3}$ is situated at the superior plane. The axes $x_{1}$ and $x_{3}$ are contained on the superior plane and the axis $x_{2}$ is vertical descendent. The thickness $H$ of the solid is divided into a set of $N$ layers, each , $n$, of them has a thickness $h_{n}$ as it is shown in figure 8. The partial thickness of the solid is denoted by $H_{n}=\sum_{j=1}^{j=n} h_{j}$ and the total thickness is $H=H_{N}$. Each layer $n$ is constituted by an isotropic elastic material of density $\rho^{n}$ and elastic Lamé constants $\lambda^{n}$ and $\mu^{n}$. These constants are related to the Young modulus $E^{n}$ and the Poisson ratio by the expressions

$$
\lambda^{n}=\frac{\nu^{n} E^{n}}{\left(1+\nu^{n}\right)\left(1-2 \nu^{n}\right)} \quad \text { y } \quad \mu^{n}=\frac{E^{n}}{2\left(1+\nu^{n}\right)}
$$

In the analysis of the wave propagation trough the solid the following variables values are: Material properties, i.e. for each layer $n$ the thickness $h_{n}$ and the elastic constants $\lambda^{n}$ and $\mu^{n}$ are known, as well as the density $\rho^{n}$. Therefore the wave propagation longitudinal $v_{L}^{n}$ and transversal $v_{T}^{n}$ velocities are also data. 


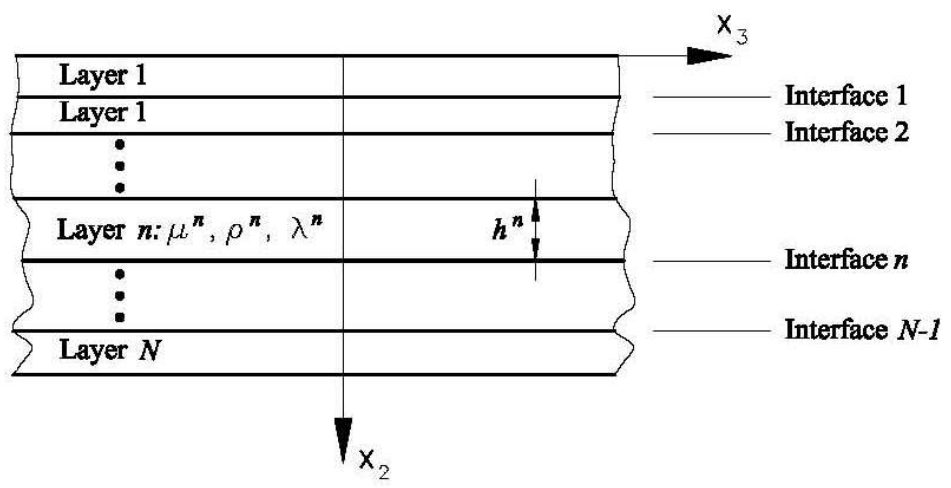

Figure 8: N-layered elastic solid

The problem to be solved consists in to compute the wave number $k$ corresponding to each specified frequency value $\omega$. The resultant transcendental equation, relating $k$ and $\omega$, is known as dispersion equation and it is derived from the condition of existence of nontrivial solution of a system of $4 \mathrm{~N}$ simultaneous equations.

\subsection{General equations}

The dynamic equilibrium equations of layer $n$ are

$$
\mu^{n} \nabla^{2} \mathbf{U}^{n}+\left(\lambda^{n}+\mu^{n}\right) \nabla\left(\nabla \cdot \mathbf{U}^{n}\right)=\rho^{n} \frac{\partial^{2} \mathbf{U}^{n}}{\partial t^{2}}
$$

and the stationary harmonic solution $\mathbf{u}(\mathbf{x})$, with $\mathbf{x}=\left(x_{1}, x_{1}, x_{1}\right)$, can be found assuming $\mathbf{U}(\mathbf{x}, t)=\mathbf{u}(\mathbf{x}) e^{i \omega t}$ se escribe:

$$
\mu^{n} \nabla^{2} \mathbf{u}^{n}+\left(\lambda^{n}+\mu^{n}\right) \nabla\left(\nabla \cdot \mathbf{u}^{n}\right)=\rho^{n} \omega^{2} \mathbf{u}^{n}
$$

If Helmholtz decomposition is introduced

$$
\mathbf{u}^{n}=\nabla \cdot \phi^{n}+\nabla \times \boldsymbol{\psi}^{n}
$$

and in each layer is assumed the displacement $u_{1}^{n}=0$ and the other displacement components $u_{2}^{n}$ and $u_{3}^{n}$ are dependent only on $x_{2}$ and $x_{3}$, i.e. the following conditions are fulfilled:

$$
\phi^{n}=\phi^{n}\left(x_{2}, x_{3}\right), \quad \psi_{2}^{n}=\psi_{3}^{n}=0 \quad \text { and } \quad \psi_{1}^{n}=\psi^{n}\left(x_{2}, x_{3}\right)
$$


then the following result is reached

$$
u_{1}^{n}=0, \quad u_{2}^{n}=\phi_{, 2}^{n}+\psi_{, 3}^{n} \quad \text { y } \quad u_{3}^{n}=\phi_{, 3}^{n}-\psi_{, 2}^{n}
$$

Introducing equations (21) into (17) the well known uncoupled equations for longitudinal and transversal wave propagation are obtained:

$$
\left[\nabla^{2}-\frac{1}{\left(v_{L}^{n}\right)^{2}} \frac{\partial^{2}}{\partial t^{2}}\right] \phi^{n}=0, \quad\left[\nabla^{2}-\frac{1}{\left(v_{T}^{n}\right)^{2}} \frac{\partial^{2}}{\partial t^{2}}\right] \psi^{n}=0
$$

in which

$$
\nabla^{2} \equiv \frac{\partial^{2}}{\partial x_{2}^{2}}+\frac{\partial^{2}}{\partial x_{3}^{2}}
$$

and the longitudinal velocity $v_{L}^{n}$ and the transversal velocity $v_{T}^{n}$ are given by the following expressions:

$$
v_{L}^{n}=\sqrt{\frac{\lambda^{n}+2 \mu^{n}}{\rho^{n}}} \quad \text { y } \quad v_{T}^{n}=\sqrt{\frac{\mu^{n}}{\rho^{n}}}
$$

\subsection{Solution}

The solution of equation (17) is

$$
\begin{aligned}
\phi^{n}= & C_{1}^{n} \exp \left[i k_{L}^{n}\left(x_{3} \operatorname{sen} \theta_{L}^{n}+x_{2} \cos \theta_{L}^{n}\right)\right] \\
& +C_{2}^{n} \exp \left[i k_{L}^{n}\left(x_{3} \operatorname{sen} \theta_{L}^{n}-x_{2} \cos \theta_{T}^{n}\right)\right] \\
\psi^{n}= & C_{3}^{n} \exp \left[i k_{T}^{n}\left(x_{3} \operatorname{sen} \theta_{T}^{n}+x_{2} \cos \theta_{T}^{n}\right)\right] \\
& +C_{4}^{n} \exp \left[i k_{T}^{n}\left(x_{3} \operatorname{sen} \theta_{T}^{n}-x_{2} \cos \theta_{T}^{n}\right)\right]
\end{aligned}
$$

with $i=\sqrt{-1}, k_{L}^{n}=\frac{\omega}{v_{L}^{n}}$ y $k_{T}^{n}=\frac{\omega}{v_{T}^{n}}$.

Each expression, (23) and (24), is a sum of two terms, one representing a downward propagating plane wave and the other representing an upward propagating term, according to the sign of the exponential term $x_{2}$ positive or negative. Each term of the former expressions are named partial waves and they are represented in figure

The values of the arbitrary constants $C_{i}^{n}$ with $i=1,2,3,4$ are found from the boundary conditions at $x_{3}=0$ and $x_{3}=H$ in which $H=\sum_{n=1}^{N} h_{n}$ and as well from the continuity conditions between the $N-1$ layer interfaces.

\subsection{Displacements}

The displacement expressions can be simplified if the following notation is introduced:

$$
J_{L}^{n}=k_{L} \operatorname{sen} \theta_{L}^{n}, \quad K_{L}^{n}=k_{L} \cos \theta_{L}^{n}, \quad J_{T}^{n}=k_{T} \operatorname{sen} \theta_{L}^{n}, \quad K_{T}^{n}=k_{T} \cos \theta_{L}^{n}
$$




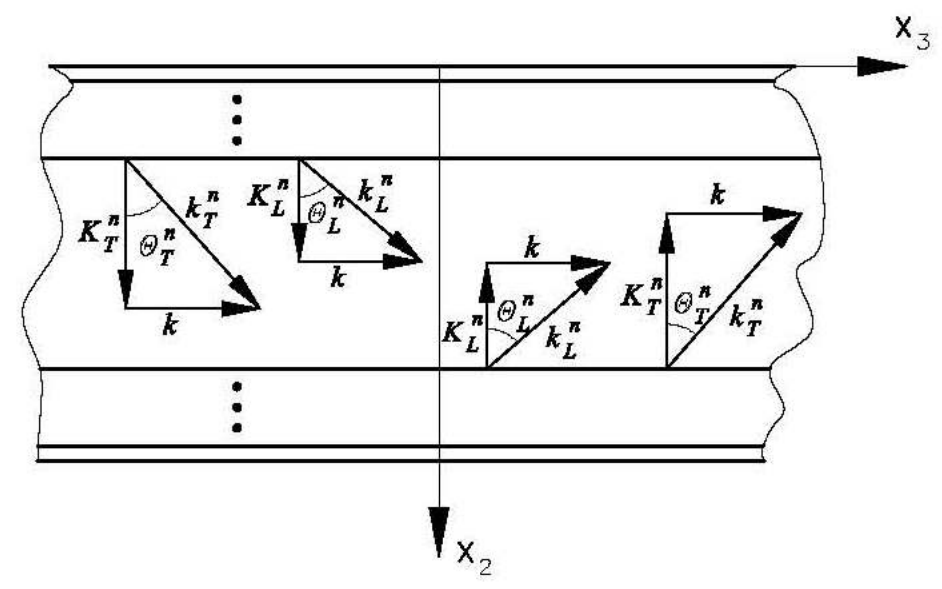

Figure 9: Four partial waves in layer $n$

Substituting (23) and (24) into (19) the following expression are reached:

$$
\begin{aligned}
u_{2}^{n}= & i K_{L}^{n}\left[C_{1}^{n} \exp \left(i J_{L}^{n} x_{3}+i K_{L}^{n} x_{2}\right)-C_{2}^{n} \exp \left(i J_{L}^{n} x_{3}-i K_{L}^{n} x_{2}\right)\right] \\
& +i J_{T}^{n}\left[C_{3}^{n} \exp \left(i J_{T}^{n} x_{3}+i K_{T}^{n} x_{2}\right)+C_{4}^{n} \exp \left(i J_{T}^{n} x_{3}-i K_{T}^{n} x_{2}\right)\right] \\
u_{3}^{n}= & i J_{L}^{n}\left[C_{1}^{n} \exp \left(i J_{L}^{n} x_{3}+i K_{L}^{n} x_{2}\right)+C_{2}^{n} \exp \left(i J_{L}^{n} x_{3}-i K_{L}^{n} x_{2}\right)\right] \\
& +i K_{T}^{n}\left[C_{3}^{n} \exp \left(i J_{T}^{n} x_{3}+i K_{T}^{n} x_{2}\right)-C_{4}^{n} \exp \left(i J_{T}^{n} x_{3}-i K_{T}^{n} x_{2}\right)\right]
\end{aligned}
$$

\subsection{Strains}

The strains are found by using the following formulae:

$$
\varepsilon=\frac{1}{2}\left(u_{i, j}+u_{j, i}\right)
$$


and if the expressions (25) and (26) are considered then the following relations are reached:

$$
\begin{aligned}
\varepsilon_{22}^{n}= & -\left(K_{L}^{n}\right)^{2}\left[C_{1}^{n} \exp \left(i J_{L}^{n} x_{3}+i K_{L}^{n} x_{2}\right)+C_{2}^{n} \exp \left(i J_{L}^{n} x_{3}-i K_{L}^{n} x_{2}\right)\right] \\
& -J_{T}^{n} K_{T}^{n}\left[C_{3}^{n} \exp \left(i J_{T}^{n} x_{3}+i K_{T}^{n} x_{2}\right)-C_{4}^{n} \exp \left(i J_{T}^{n} x_{3}-i K_{T}^{n} x_{2}\right)\right] \\
\varepsilon_{33}^{n}= & -\left(J_{L}^{n}\right)^{2}\left[C_{1}^{n} \exp \left(i J_{L}^{n} x_{3}+i K_{L}^{n} x_{2}\right)+C_{2}^{n} \exp \left(i J_{L}^{n} x_{3}-i K_{L}^{n} x_{2}\right)\right] \\
& +J_{T}^{n} K_{T}^{n}\left[C_{3}^{n} \exp \left(i J_{T}^{n} x_{3}+i K_{T}^{n} x_{2}\right)-C_{4}^{n} \exp \left(i J_{T}^{n} x_{3}-i K_{T}^{n} x_{2}\right)\right] \\
\varepsilon_{23}^{n}= & -J_{L}^{n} K_{L}^{n}\left[C_{1}^{n} \exp \left(i J_{L}^{n} x_{3}+i K_{L}^{n} x_{2}\right)-C_{2}^{n} \exp \left(i J_{L}^{n} x_{3}-i K_{L}^{n} x_{2}\right)\right] \\
& +\frac{\left(K_{T}^{n}\right)^{2}-\left(J_{T}^{n}\right)^{2}}{2}\left[C_{3}^{n} \exp \left(i J_{T}^{n} x_{3}+i K_{T}^{n} x_{2}\right)\right. \\
& \left.+C_{4}^{n} \exp \left(i J_{T}^{n} x_{3}-i K_{T}^{n} x_{2}\right)\right]
\end{aligned}
$$

It is convenient to obtain the expression of the dilation deformation Es conveniente obtener la expresión de la deformación volumétrica definida $e^{n}$ defined as follows: $e^{n}=\varepsilon_{11}^{n}+\varepsilon_{22}^{n}=\nabla \mathbf{u}^{n}$, i.e.

$$
\begin{aligned}
e & =\nabla \mathbf{u}^{n}=\nabla\left(\nabla \cdot \phi^{n}+\nabla \times \boldsymbol{\psi}^{n}\right)=\nabla^{2} \phi^{n}=-\left(k_{L}^{n}\right)^{2} \phi^{n} \\
& =-\left(k_{L}^{n}\right)^{2}\left[C_{1}^{n} \exp \left(i J_{L}^{n} x_{3}+i K_{L}^{n} x_{2}\right)+C_{2}^{n} \exp \left(i J_{L}^{n} x_{3}-i K_{L}^{n} x_{2}\right)\right]
\end{aligned}
$$

\subsection{Stresses}

The stresses (pressure forces) acting on the faces of each layer are found from the Lamé constitutive equations as follows:

$$
\sigma_{i j}^{n}=\lambda^{n} e^{n} \delta_{i j}+2 \mu^{n} \varepsilon_{i j}^{n}
$$

and the following result is obtained

$$
\begin{aligned}
& \sigma_{22}^{n}=\lambda^{n} e^{n}+2 \mu^{n} \varepsilon_{22}^{n}=-\left(k_{L}^{n}\right)^{2}\left[\lambda^{n}+2 \mu^{n} \cos ^{2} \theta_{L}^{n}\right] \\
& \quad\left[C_{1}^{n} \exp \left(i J_{L}^{n} x_{3}+i K_{L}^{n} x_{2}\right)+C_{2}^{n} \exp \left(i J_{L}^{n} x_{3}-i K_{L}^{n} x_{2}\right)\right] \\
& \quad-2 \mu^{n} J_{T}^{n} K_{T}^{n}\left[C_{3}^{n} \exp \left(i J_{T}^{n} x_{3}+i K_{T}^{n} x_{2}\right)-C_{4}^{n} \exp \left(i J_{T}^{n} x_{3}-i K_{T}^{n} x_{2}\right)\right] \\
& \sigma_{23}^{n}=2 \mu^{n} \varepsilon_{23}^{n}=-2 \mu^{n} J_{L}^{n} K_{L}^{n} \\
& \quad\left[C_{1}^{n} \exp \left(i J_{L}^{n} x_{3}+i K_{L}^{n} x_{2}\right)-C_{2}^{n} \exp \left(i J_{L}^{n} x_{3}-i K_{L}^{n} x_{2}\right)\right] \\
& +\mu^{n}\left[\left(J_{T}^{n}\right)^{2}-\left(K_{T}^{n}\right)^{2}\right]\left[C_{3}^{n} \exp \left(i J_{T}^{n} x_{3}+i K_{T}^{n} x_{2}\right)+C_{4}^{n} \exp \left(i J_{T}^{n} x_{3}-i K_{T}^{n} x_{2}\right)\right]
\end{aligned}
$$

\subsection{Boundary conditions}

It is assumed all the $N$ layers are solid, i.e, intermediate liquids does not exist. In the case of existence of a liquid layer the boundary conditions are represented 
by zero shear stresses and continuity of displacements and normal stresses in the interfaces between layers. The cases of existence of liquid layers can be treated by pertinent changes of the case of all solid layers, that will be described in the following section.

\subsubsection{Layer 1. Free upper face}

The free face is $\left(x_{2}=0, \quad-\infty<x_{3}<\infty\right)$ and the boundary conditions to be imposed are: $\sigma_{22}^{1}=\sigma_{23}^{1}=0$, i.e:

$$
\begin{aligned}
\sigma_{22}^{1}=0 \rightarrow & \left(k_{L}^{1}\right)^{2}\left[\lambda^{1}+2 \mu^{1} \cos ^{2} \theta_{L}^{1}\right]\left[C_{1}^{1}+C_{2}^{1}\right] \exp \left(i J_{L}^{1} x_{3}\right) \\
& +2 \mu^{1} J_{T}^{1} K_{T}^{1}\left[C_{3}^{1}-C_{4}^{1}\right] \exp \left(i J_{T}^{1} x_{3}\right)=0 \\
\sigma_{23}^{1}=0 \rightarrow & -2 \mu^{1} J_{L}^{1} K_{L}^{1}\left[C_{1}^{1}-C_{2}^{1}\right] \exp \left(i J_{L}^{1} x_{3}\right) \\
& +\mu^{1}\left[\left(J_{T}^{1}\right)^{2}-\left(K_{T}^{1}\right)^{2}\right]\left[C_{3}^{1}+C_{4}^{1}\right] \exp \left(i J_{T}^{1} x_{3}\right)=0
\end{aligned}
$$

In order all the former equations became null it is necessary the following condition is satisfied for all values of $x_{3}$, i.e., $J_{L}^{1}=J_{T}^{1}$. This condition implies $k_{L}^{1} \operatorname{sen} \theta_{L}^{1}=k_{T}^{1} \operatorname{sen} \theta_{T}^{1}=k$. This relationship represents the reflection law of Snell. It can be shown the validity of this condition for all values of $n$ non equal to 1 , therefore the reflection law of Snell can be written in general as follows $k_{L}^{n} \operatorname{sen} \theta_{L}^{n}=k_{T}^{n} \operatorname{sen} \theta_{T}^{n}=k$. It can be observed the coefficients $J_{L}^{n}, K_{L}^{n}, J_{T}^{n}$ and $K_{T}^{n}$ can be expresses as a function of the wave number $k$ as follows:

$$
\begin{array}{ll}
J_{L}^{n}=k_{L}^{n} \operatorname{sen} \theta_{L}^{n}=k, & J_{T}^{n}=k_{T}^{n} \operatorname{sen} \theta_{T}^{n}=k \\
K_{L}^{n}=k_{L}^{n} \cos \theta_{L}^{n}=\sqrt{\left(k_{L}^{n}\right)^{2}-k^{2},} & K_{T}^{n}=k_{T}^{n} \operatorname{sen} \theta_{T}^{n}=\sqrt{\left(k_{T}^{n}\right)^{2}-k^{2}}
\end{array}
$$

In the case of $k^{2}<\left(k_{L}^{n}\right)^{2}$ the positive roor is adopted, i.e. $K_{L}^{n}=\sqrt{\left(k_{L}^{n}\right)^{2}-k^{2}}$. By the contrary if $k^{2}>\left(k_{L}^{n}\right)^{2}$ then $K_{L}^{n}=i \sqrt{\left(k^{2}-k_{L}^{n}\right)^{2}}$. Similar results are applied for the expression of the transversal wave $K_{T}^{n}$.

Then, the equations (34) and (35) can be written in the form:

$$
\begin{aligned}
& {\left[\lambda^{1}\left(k_{L}^{1}\right)^{2}+2 \mu^{1}\left(K_{L}^{1}\right)^{2}\right]\left[C_{1}^{1}+C_{2}^{1}\right]+2 \mu^{1} k K_{T}^{1}\left[C_{3}^{1}-C_{4}^{1}\right]=0} \\
& -2 k \mu^{1} K_{L}^{1}\left[C_{1}^{1}-C_{2}^{1}\right]+\mu^{1}\left[\left(K_{T}^{1}\right)^{2}-k^{2}\right]\left[C_{3}^{1}+C_{4}^{1}\right]=0
\end{aligned}
$$

\subsubsection{Layers $n$ and $n+1$. Interface $n$}

The interface $n$ is defined as $\left(x_{2}=H_{n}, \quad-\infty<x_{3}<\infty\right)$ with $H_{n}=\sum_{j=1}^{n} h_{j}$ and the conditions to be imposed are: $u_{2}^{n}=u_{2}^{n+1}, u_{3}^{n}=u_{3}^{n+1}, \sigma_{22}^{n}=\sigma_{22}^{n+1}$ 
$\sigma_{23}^{n}=\sigma_{23}^{n+1}, \operatorname{con} n=1,2, \ldots, N-1$ i.e.:

$u_{2}^{n}=u_{2}^{n+1}$ implica:

$K_{L}^{n}\left[C_{1}^{n} \exp \left(i K_{L}^{n} H_{n}\right)-C_{2}^{n} \exp \left(-i K_{L}^{n} H_{n}\right)\right]$

$+k\left[C_{3}^{n} \exp \left(i K_{T}^{n} H_{n}\right)+C_{4}^{n} \exp \left(-i K_{T}^{n} H_{n}\right)\right]$

$-K_{L}^{n+1}\left[C_{1}^{n+1} \exp \left(i K_{L}^{n+1} H_{n}\right)-C_{2}^{n+1} \exp \left(-i K_{L}^{n+1} H_{n}\right)\right]$

$-k\left[C_{3}^{n+1} \exp \left(i K_{T}^{n+1} H_{n}\right)+C_{4}^{n+1} \exp \left(-i K_{T}^{n+1} H_{n}\right)\right]=0$

$u_{3}^{n}=u_{3}^{n+1}$ implica:

$k\left[C_{1}^{n} \exp \left(i K_{L}^{n} H_{n}\right)+C_{2}^{n} \exp \left(-i K_{L}^{n} H_{n}\right)\right]$

$-K_{T}^{n}\left[C_{3}^{n} \exp \left(i K_{T}^{n} H_{n}\right)-C_{4}^{n} \exp \left(-i K_{T}^{n} H_{n}\right)\right]$

$-k\left[C_{1}^{n+1} \exp \left(i K_{L}^{n+1} H_{n}\right)+C_{2}^{n+1} \exp \left(-i K_{L}^{n+1} H_{n}\right)\right]$

$+K_{T}^{n+1}\left[C_{3}^{n+1} \exp \left(i K_{T}^{n+1} H_{n}\right)-C_{4}^{n+1} \exp \left(-i K_{T}^{n+1} H_{n}\right)\right]=0$

$\sigma_{22}^{n}=\sigma_{22}^{n+1}$ implica:

$\left[\lambda^{n}\left(k_{L}^{n}\right)^{2}+2 \mu^{n}\left(K_{L}^{n}\right)^{2}\right]\left[C_{1}^{n} \exp \left(i K_{L}^{n} H_{n}\right)+C_{2}^{n} \exp \left(-i K_{L}^{n} H_{n}\right)\right]$

$+2 \mu^{n} k K_{T}^{n}\left[C_{3}^{n} \exp \left(i K_{T}^{n} H_{n}\right)-C_{4}^{n} \exp \left(-i K_{T}^{n} H_{n}\right)\right]$

$-\left[\lambda^{n+1}\left(k_{L}^{n+1}\right)^{2}+2 \mu^{n+1}\left(K_{L}^{n+1}\right)^{2}\right]\left[C_{1}^{n+1} \exp \left(i K_{L}^{n+1} H_{n}\right)+C_{2}^{n+1} \exp \left(-i K_{L}^{n+1} H_{n}\right)\right]$

$+2 \mu^{n+1} k K_{T}^{n+1}\left[C_{3}^{n+1} \exp \left(i K_{T}^{n+1} H_{n}\right)-C_{4}^{n+1} \exp \left(-i K_{T}^{n+1} H_{n}\right)\right]=0$

$\sigma_{23}^{n}=\sigma_{23}^{n+1}$ implica:

$-2 k \mu^{n} K_{L}^{n}\left[C_{1}^{n} \exp \left(i K_{L}^{n} H_{n}\right)-C_{2}^{n} \exp \left(-i K_{L}^{n} H_{n}\right)\right]$

$+\mu^{n}\left[\left(K_{T}^{n}\right)^{2}-k^{2}\right]\left[C_{3}^{n} \exp \left(i K_{T}^{n} H_{n}\right)+C_{4}^{n} \exp \left(-i K_{T}^{n} H_{n}\right)\right]$

$+2 k \mu^{n} K_{L}^{n+1}\left[C_{1}^{n+1} \exp \left(i K_{L}^{n+1} H_{n}\right)-C_{2}^{n+1} \exp \left(-i K_{L}^{n+1} H_{n}\right)\right]$

$-\mu^{n+1}\left[\left(K_{T}^{n+1}\right)^{2}-k^{2}\right]\left[C_{3}^{n+1} \exp \left(i K_{T}^{n+1} H_{n}\right)+C_{4}^{n+1} \exp \left(-i K_{T}^{n+1} H_{n}\right)\right]=0$

\subsubsection{Capa $N$. Free lower face}

The lower free surface is given by the expression $\left(x_{2}=H, \quad-\infty<x_{3}<\infty\right)$ and the boundary conditions to be imposed are: $\sigma_{22}^{N}=\sigma_{23}^{N}=0$, i.e.:

$$
\begin{aligned}
\sigma_{22}^{N}=0 \rightarrow & {\left[\lambda^{N}\left(k_{L}^{N}\right)^{2}+2 \mu^{N}\left(K_{L}^{N}\right)^{2}\right]\left[C_{1}^{N} \exp \left(i K_{L}^{N} H\right)+C_{2}^{N} \exp \left(-i K_{L}^{N} H\right)\right] } \\
& +2 \mu^{N} k K_{T}^{N}\left[C_{3}^{N} \exp \left(i K_{T}^{N} H\right)-C_{4}^{N} \exp \left(-i K_{T}^{N} H\right)\right]=0 \\
\sigma_{23}^{N}=0 \rightarrow & -2 k \mu^{N} K_{L}^{N}\left[C_{1}^{N} \exp \left(i K_{L}^{N} H\right)-C_{2}^{N} \exp \left(-i K_{L}^{N} H\right)\right] \\
& +\mu^{N}\left[\left(K_{T}^{N}\right)^{2}-k^{2}\right]\left[C_{3}^{N} \exp \left(i K_{T}^{N} H\right)+C_{4}^{N} \exp \left(-i K_{T}^{N} H\right)\right]
\end{aligned}
$$




\subsection{Capa $N$ de espesor infinito}

In some situations, particularly in problems of identification of layered solids, it is assumed the depth of the base rock is very large. Then the boundary condition of the former section, corresponding to the lower face of the layer $N$ can be substitute by other special one, that will be commented in the following. For very large values of $x_{2}$ the wave becomes null and then the constant $K_{L}^{N}$ must be an imaginary number. Therefore, the boundary conditions $\sigma_{22}^{N}=\sigma_{23}^{N}=0$ at $x_{2} \rightarrow \infty$ are expressed according to the following equations:

$$
C_{2}=0 \quad \text { and } \quad C_{4}=0
$$

\subsection{Dispersion equation}

By introducing the following notation

$$
\mathbf{C}^{n}=\left(\begin{array}{cccc}
C_{1}^{n} & C_{2}^{n} & C_{3}^{n} & C_{4}^{n}
\end{array}\right)^{T}
$$

the former boundary and continuity conditions can be written in matrix form:

$$
\begin{aligned}
& \mathbf{A}_{11}^{0} \mathbf{C}^{1}=\mathbf{0} \\
& \mathbf{A}_{11} \mathbf{C}^{1}+\mathbf{A}_{12} \mathbf{C}^{2}=\mathbf{0} \\
& \mathbf{A}_{22} \mathbf{C}^{2}+\mathbf{A}_{23} \mathbf{C}^{3}=\mathbf{0} \\
& \cdots \\
& \mathbf{A}_{n, n} \mathbf{C}^{n}+\mathbf{A}_{n, n+1} \mathbf{C}^{n+1}=\mathbf{0} \\
& \mathbf{A}_{n+1, n+1} \mathbf{C}^{n+1}+\mathbf{A}_{n+1, n+2} \mathbf{C}^{n+2}=\mathbf{0} \\
& \cdots \\
& \mathbf{A}_{N-1, N-1} \mathbf{C}^{N-1}+\mathbf{A}_{N-1, N} \mathbf{C}^{N}=\mathbf{0} \\
& \mathbf{A}_{N, N}^{0} \mathbf{C}^{N}=\mathbf{0}
\end{aligned}
$$

with $\mathbf{A}_{11}^{0}$ and $\mathbf{A}_{N, N}^{0}$ coefficients matrices with $2 \times 4$ dimension. The dimension of coefficients matrices $\mathbf{A}_{n, n}$ and $\mathbf{A}_{n, n+1}$ is $4 \times 4$. All th elements of these matrices are functions of the problem data. The unique unknown to be found, for each specified frequency $\omega$, is the wave number $k$. The remaining variables of the coefficients of the formar matrices can be expressed as functions of the unknown $k$, according to equations (36) and (37), en función de la incógnita.

The number of unknowns of the system of homogenous equations (51) is 2 for each end surface (lower and upper faces) and 4 for each interface, then the total number of unknowns is $2 \times 2+4(N-1)=4 N$ plus four constants $C_{i}^{n}$ for each 
layer $n$, i.e. the total number is $4 N$. Therefore the dimension of the system (51) is $4 N \times 4 N$ and it can be written:

$$
\mathrm{AC}=0
$$

in which $\mathbf{C}=\left(\mathbf{C}^{1 T}, \mathbf{C}^{2 T}, \ldots, \mathbf{C}^{N T}\right)^{T}$ and the coefficients matrix $\mathbf{A}$ is given by the expression:

$$
\mathbf{A}=\left[\begin{array}{ccccccccc}
\mathbf{A}_{11}^{0} & \mathbf{0} & \mathbf{0} & \mathbf{0} & . & . & \mathbf{0} & \mathbf{0} & \mathbf{0} \\
\mathbf{A}_{11} & \mathbf{A}_{12} & \mathbf{0} & \mathbf{0} & . & . & \mathbf{0} & \mathbf{0} & \mathbf{0} \\
\mathbf{0} & \mathbf{A}_{22} & \mathbf{A}_{23} & \mathbf{0} & . & . & \mathbf{0} & \mathbf{0} & \mathbf{0} \\
\mathbf{0} & \mathbf{0} & \mathbf{0} & \mathbf{0} & . & . & . & . & . \\
\mathbf{0} & \mathbf{0} & \mathbf{0} & \mathbf{A}_{n, n} & \mathbf{A}_{n, n+1} & \mathbf{0} & \mathbf{0} & \mathbf{0} & \mathbf{0} \\
\mathbf{0} & \mathbf{0} & \mathbf{0} & \mathbf{0} & \mathbf{A}_{n+1, n+1} & \mathbf{A}_{n+1, n+2} & \mathbf{0} & \mathbf{0} & \mathbf{0} \\
\mathbf{0} & \mathbf{0} & \mathbf{0} & \mathbf{0} & . & . & \mathbf{0} & \mathbf{0} & \mathbf{0} \\
. & . & . & . & . & . & . & \mathbf{A}_{N-1, N-1} & \mathbf{A}_{N-1, N} \\
\mathbf{0} & \mathbf{0} & \mathbf{0} & \mathbf{0} & . & . & \mathbf{0} & \mathbf{0} & \mathbf{A}_{N, N}^{0}
\end{array}\right]
$$

In order the solution of the system of homogenous equations (51) is non trivial it is necessary that the the determinant of matrix $\mathbf{A}$ is zero, i. e. the following dispersion equation must be satisfied:

$$
\operatorname{det}(\mathbf{A})=0, \quad \text { es } \operatorname{decir}\left|\mathbf{A}\left(\omega, k, \lambda^{n}, \mu^{n}, h_{n}\right)\right|=0
$$

In equation (53) the data are the constants $\lambda^{n}, \mu^{n}, h_{n}$ for each layer $n$, with $n=$ $1,2, \ldots, N$. The wave numbers $K_{L}^{n}$ and $K_{T}^{n}$ can be expressed as function of the unknowns $k$ and $\omega$, according to formulae (36) and (37). Then by solving equation (53) it is possible to compute for each frequency $\omega$ the infinite values of $k$, although a finite number of $k$ are real values, i.e. non imaginary numbers. Each par of solutions defines the phase velocity $c_{p}=\frac{\omega}{k}$.

Several numerical procedures exist to find solutions $k$ for each values of $\omega$ and some of them will be discussed in a following section.

\subsection{Results}

By sweeping of the pair $k$ and $\omega$ it is possible to represent the spectrum of frequency. In addition the dispersion curves defined by $c_{p}=\frac{\omega}{k}$ as function of the frequency $\omega$ is another result of interest.

Once the pair of values, $k_{j}$ and $\omega_{j}$, have been obtained as solution of the dispersion equation (53) it is necessary to compute the values of the constantes $\mathbf{C}=\mathbf{C}_{j}$, 
assuming the system of equations particularized for the values $k_{j}$ and $\omega_{j}$, i.e.,

$$
\mathbf{C}_{j}=\left[\begin{array}{c}
\mathbf{C}_{j}^{1} \\
\mathbf{C}_{j}^{2} \\
\cdot \\
\mathbf{C}_{j}^{n} \\
\cdot \\
\mathbf{C}_{j}^{N}
\end{array}\right] \quad \text { with } \quad \mathbf{C}_{j}^{n}=\left[\begin{array}{c}
\mathbf{C}_{j 1}^{n} \\
\mathbf{C}_{j 2}^{n} \\
\mathbf{C}_{j 3}^{n} \\
\mathbf{C}_{j 4}^{n}
\end{array}\right]
$$

From the knowledge of the values of these constants the following results of interest can be found as follows:

- Displacement values $u_{1}\left(x_{2}, x_{3}\right)$ and $u_{2}\left(x_{2}, x_{3}\right)$ as function of $x_{2}$ by application of expressions (25) and (26).

- Values of the strains $\varepsilon_{22}\left(x_{2}, x_{3}\right), \varepsilon_{33}\left(x_{2}, x_{3}\right)$ and $\varepsilon_{23}\left(x_{2}, x_{3}\right)$ as function of $x_{2}$, where $x_{3}$ and $t$ are parameters. They can be computed by the formulae (27), (28) and (29).

- Values of the stresses $\sigma_{22}\left(x_{2}, x_{3}\right), \sigma_{33}\left(x_{2}, x_{3}\right)$ and $\sigma_{23}\left(x_{2}, x_{3}\right)$ as function of $x_{2}$, where $x_{3}$ and $t$ are parameters. They can be found by using formulaes

$$
\begin{aligned}
& \sigma_{22}^{n}=\lambda^{n} e^{n}+2 \mu^{n} \varepsilon_{22}^{n} \\
& \sigma_{33}^{n}=\lambda^{n} e^{n}+2 \mu^{n} \varepsilon_{33}^{n} \\
& \sigma_{23}^{n}=2 \mu^{n} \varepsilon_{23}^{n}
\end{aligned}
$$

in which $e$ is the volumetric deformation given by the expression (30).

\section{Numerical solution of the dispersion curve}

A general numerical procedure has been developed to build up the matriz $\mathbf{A}$ of complex elements, to solve the nonlinear equation (53) and generate the dispersion curve $c_{p}=\frac{\omega}{k}$.

Algorithms with global convergence, i.e. aimed to obtain an approximate value of the root are used for an initial guess of an interval of approximated solutions [1]. Among them it have been used the bisection procedure (if zero simples are considered) and the algorithm based on the change of slope detection and minimum value of the function $\operatorname{det}(\mathbf{A})$ (if multiple zeros are taken in consideration). The obtained values are then starting values for the local convergence algorithm. Due to the difficulty to obtain an explicit derivative of the function a method of linear interpolation has been selected using the secant procedure. The convergence order in this case for simple roots has been 1.618 [8] but this for multiple 
roots the convergence order is deteriorated to become of order 1 . However in this last case the Aitken convergence acceleration technique [5] can be applied. It has also been tested also the algorithm of quadratic inverse interpolation (method of Muller) with similar convergence results computational but more efficient than the Aitken method although this method demands three initial values to be applied.

Using the programming environment Matlab several subroutines have been written.

The main computational steps of this analysis are summarized as follows:

1. Computation of Lamb modes. For a given frequency, within the interval $(k 1, k 2)$ the graph $\operatorname{det}(A)-k$ is represented and the zeros of the dispersion equation can be computed. En el caso de simple zeros the bisection algorithm is efficient to obtain a preliminar approximation of the root. In case of a multiple zero it is necessary to detect a slope change and also the minimum of the absolute value of the function $\operatorname{det} \mathbf{A}$ in order to find an approximation of the root.

2. The selection of the propagation mode of interest. The mode is selected by choosing a value close, obtained already in the step 1, to the exact solution either for $k$ (or $c_{p}$ ) and this value is used as a starting point for the generation program (program of local convergence) of the dispersion curve. In this case the secant method, or a algorithm based on quadratic inverse interpolation (Muller, Dekker-Brent) can be used.

\section{Results validation}

The validation example is the simulation of a Lamb wave propagation through a plate of total thickness $h$ considering this plate as a composite material composed by $N$ layers of identical thickness $\frac{h}{N}$ and equal properties.

To this end the following example already studied in a previous section will analyzed.

A rectangular steel plate with the following dimensions. Total thickness $2 h=$ $0.02 \mathrm{~m}$, plan dimension are $a=0.200 \mathrm{~m}$ and $b=0.120 \mathrm{~m}$. A train of symmetric Lamb waves is introduced trough the side of length $a$ ( $\alpha=0$ and frequency $f=$ $200 \times 10^{3}$. The properties of the plate material are: Young modulus $E=1.962 \times$ $10^{8} \mathrm{MPa}$, Poisson ratio $\nu=0.3093$ and density $\rho=7.797 \mathrm{t} / \mathrm{m}^{3}$. From these data is obtained the angular frequency is $\omega=1256637.061 \mathrm{rad} / \mathrm{s}$, the transversal and longitudinal velocities: $v_{T}=3099.9248 \mathrm{~m} / \mathrm{s}$ and $v_{L}=5889.5724 \mathrm{~m} / \mathrm{s}$. The dispersion equation (8) the these values can be used to find the wave number $k=218.1658 \mathrm{~m}^{-1}$ and the wave velocity $V=5760.01 \mathrm{~m} / \mathrm{sec}$. In this particular case, the Lamb wave is located in the region 2, i.e. $p$ is an imaginary number and 
$q$ is a real number. For comparative purpose the plan dimensions of the plate are supposed to be very large in order to simulate a plane strain as is assumed in the Lamb wave propagation.

It has been modeled the former plate as a N-layered plate, with $N=2,3,4$ and 5 layers and the analysis of the wave propagation trough the multi-layer plate has given the following results for the wave number that coincide with the ones found using the dispersion equation (8), as is shown in the following array:

$$
\begin{array}{rrrrr}
n= & 2 & 3 & 4 & 5 \\
k= & 218.1658 & 218.1658 & 218.1658 & 218.1658
\end{array}
$$

The displacements

$$
u_{j}\left(x_{1}, x_{2}, t\right)=u_{j}\left(x_{2}\right) \exp i\left(\omega t-k x_{1}\right) \quad \text { with } \quad j=1,2
$$

of the plate subjected to Lamb waves are computed according to expressions (12) and (13), assuming they are applied for the region 2 . These displacements are compared with the ones obtained using the expressions

$$
u_{j}\left(x_{1}, x_{2}, t\right)=u_{j}\left(x_{1}, x_{2}\right) \exp i \omega t \quad \text { with } \quad j=1,2
$$

in which the displacements $u_{j}\left(x_{1}, x_{2}\right)$ are found from the analysis of multi-layered elements, once the constants $\mathbf{C}$ are computed, using formulae (25) and (26) for the region 2 .

The following dispersion curves obtained for the former plate modeled as a N-layered plate with $N=2,3$ and 4 are shown in the following figures

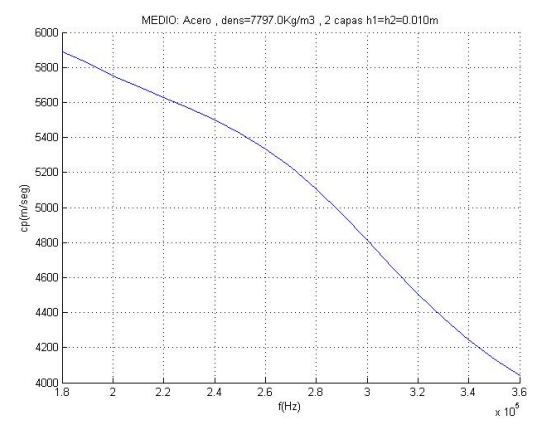

Figure 10: 2-layered elastic solid 

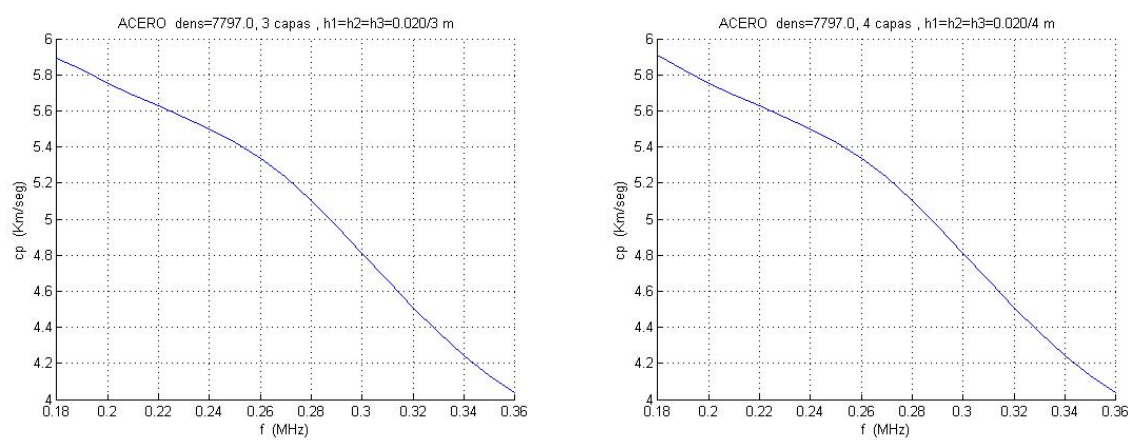

Figure 11: (a) 3-layered elastic solid. (b) 4-layered elastic solid

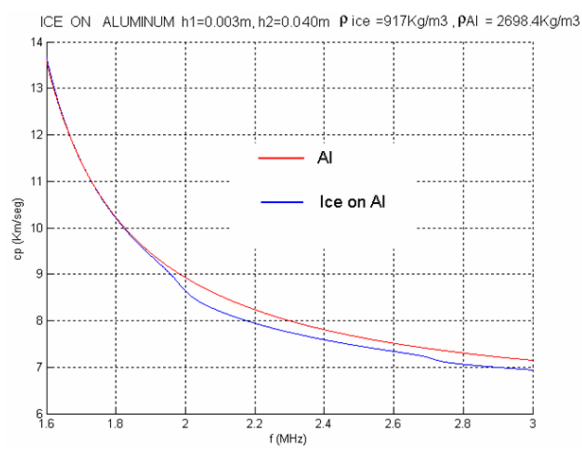

Figure 12: Ejemplo de Cardona 1

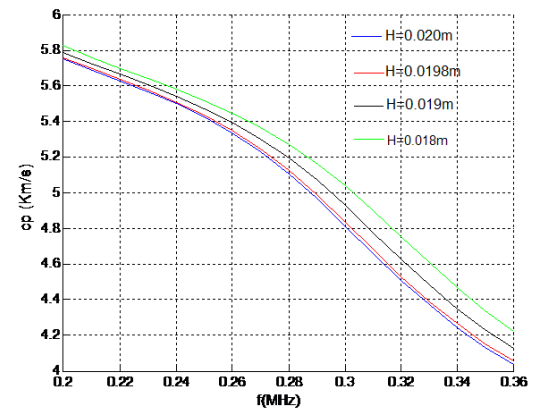

Figure 13: Ejemplo de Cardona 2 


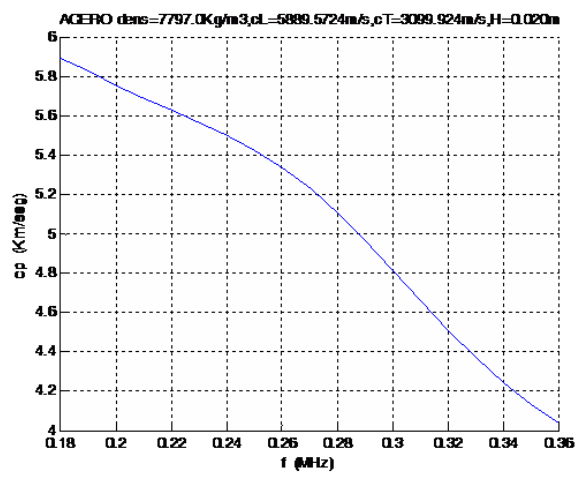

Figure 14: Ejemplo de Cardona 3

\section{Applications. Detection of imperfections}

Here imperfection means any modification of the data assumed in the analytical model or in the design of the multi-layered plate. These imperfections can be related either with model geometry (thicknesses $h_{n}$ ) or mechanical constants $E^{n}$ or $\nu^{n}$. Also the existence of cracks or micro-cracks in the external faces or inside of the plate is considered an imperfection.

The following technique for identification and possible localization of imperfections in a N-layered plate is proposed:

1. An analysis of the wave propagation trough a perfect, i.e. without imperfections, N-layered plate is carried out according to the analysis procedure described in a former section. In this analysis the following results are computed,displacements $u_{1}\left(x_{2}, x_{3}\right) \exp (i \omega t)$ and $u_{2}\left(x_{2} x_{3}\right) \exp (i \omega t)$ for a set of values of $k$ and $\omega$, satisfying the dispersion equation.

2. A laboratory dynamic test of a sample of the N-layered plate with the potential imperfection to be detected is carried out.

3. The former test is mathematically simulated by a numerical model according to the following steps:

- The tested sample with the assumed imperfection, geometry and properties is modeled using a Finite Element (FE) model. This model is typically two-dimensional (plane strain). The boundary conditions along the plate border $x_{3}=0$ are displacements $u_{2}$ and $u_{3}$ constraint. In this dynamic analysis a commercial software on FE, as ANSYS, SAP o ABAQUS, can be used. 
- This model with imperfection is analyzed under the following dynamic distributed loading

- Load along direction $x_{2}: f_{I 2}\left(x_{2}, x_{3}, t\right)=\varrho \omega^{2} u_{2}\left(0, x_{3}\right) \exp (i \omega t)$

- Load along direction $x_{3}: f_{I 3}\left(x_{2}, x_{3}, t\right)=\varrho \omega^{2} u_{3}\left(0, x_{3}\right) \exp (i \omega t)$

The analysis can be carried out either the modal superposition or using a scheme explicit or implicit in Finite Differences (Newmark, constant or linear acceleration lineal etc.). The results obtained in this analysis is called dynamic or relative response of the plate and they are written as $u_{i}\left(x_{2}, x_{3}, t\right)$ with $i=1,2$.

- The response of the tested model or total response $u_{t i}\left(x_{2}, x_{3}, t\right)$ is the sum of the dynamic response and the and the pseudo static response, defined by the expressions $u_{s i}\left(0, x_{3}, t\right)=u_{i}\left(x_{2}, x_{3}\right) \exp (i \omega t)$, i.e. (with $i=1,2$ ):

$$
u_{t i}\left(x_{2}, x_{3}, t\right)=u_{i}\left(x_{2}, x_{3}, t\right)+u_{i}\left(0, x_{3}\right) \exp (i \omega t)
$$

4. The experimental results from the test are designed by $u_{i}^{e x p}\left(x_{2}, x_{3}, t\right)$ and the theoretical and analytical results by $u_{i}^{\text {teor }}\left(x_{2}, x_{3}, t\right)$. The existence of an imperfection can be assured if the following condition is fulfilled:

$$
|O F| \leq \epsilon
$$

in which $O F=\left\|\triangle u_{i}\right\|=\left\|u_{i}^{e x p}\left(x_{2}, x_{3}, t\right)-u_{i}^{\text {teor }}\left(x_{2}, x_{3}, t\right)\right\|$, the norm $\|$. is a norm type $L_{2}$ or $L_{\infty}$ and the positive constant $\epsilon$ meaning the maximum admissible error.

For example the norm $L_{2}$ is defined by the expression

$$
\left\|\triangle u_{i}\right\|=\sum_{i=1}^{2} \int_{0}^{T_{e}} \int_{x_{2}=0}^{H} \int_{x_{3}=0}^{L_{e}}\left\|\triangle u_{i}\right\| d x_{2} d x_{3} d t
$$

5. The localization of the imperfection, defined by the parameters $z_{j}$, with $j=$ $1,2, \ldots, J$, can be reached by solving the following optimization problem

$$
\min _{z_{j}} O F
$$

The values of $z_{j}$ minimizing the objective function $O F$ allow us to identify the size and position of the imperfection. 


\section{Summary and conclusions}

1. The analysis of wave propagation in N-layered composite media is currently an important topic in relation of NDT.

2. Dispersion equations characterize a N-layered plate.

3. A dispersion equation represents a challenge due to its dimension, imaginary and real numbers involvement and existence of multiple roots.

4. Separation of roots and dispersion curves deserves special study.

\section{Acknowledgments}

The financial support for this work provided by the Dirección General de Investigación of the Spanish Ministry of Education and Science under the research contract DPI2005-09203-02 is grateful recognized by the authors.

\section{References}

[1] Forsythe, G.E. and Malcolm, M.A. and Moler, C.B. Computer Methods for Mathematical Computations. Prentice -Hall, New Jersey, 1977.

[2] Gurdal, Z. and Haftka, R.T. and Hajela, P. Design and Optimization of Laminated Composite Materials. John Wiley and Sons, Princeton, 1999.

[3] Jones, R.M. Mechanics of Composite Materials. McGraw-Hill Book Book, New York, 1975.

[4] Landau, L.D. and Lifshitz, E.M. Theory of Elasticity, volume 7 of Course of Theoretical Physics. Pergamon Press, New York, 1959.

[5] Mathews, K.D. and Fink, K.D. Numerical Methods using Matlab. Prentice Hall, New Jersey, 1998.

[6] Rose, J.L. Ultrasonic Waves in Solid Media. Cambridge University Press, Cambridge, 2004.

[7] Royer, D. and Dieulesaint, E. Elastic Waves in solids. Vols I and II. Springer Verlag, Berlin-Heidelberg, 2000.

[8] Stoer, J. and Bulirsch, R. Introduction to Numerical Analysis. Springer Verlag, New York, 1980. 
[9] Tsai, S.W. and Pagano, N.J. Composite Materials Workshop, chapter Invariant Properties of Composite Materials, pages 233-253. Technomic Oublishing Co., Westport, 1968.

[10] Vinson, J.R. and 'Sierakowski, R.L. The Behaviour of Structures Composed of Composite Materials. Nijhoff, Netherlands, 1989.

[11] Whitney, J.M. Structural Analysis of Laminate Anisotropic Plates. Technomic, 1987. 